\title{
Triamcinolone Acetonide-Loaded Nanoparticles Encapsulated By CD90+ MCSs-Derived Microvesicles Drive Anti-Inflammatory Properties and Promote Cartilage Regeneration After Osteoarthritis
}

Yuanlong Li ( $\sim$ liyuanlong2015@foxmail.com )

Jinzhou Medical University

Qingqiang Tu

Sun Yat-sen University of Medical Sciences: Sun Yat-sen University Zhongshan School of Medicine Dongmei Xie

Sun Yat-sen University of Medical Sciences: Sun Yat-sen University Zhongshan School of Medicine Shurui Chen

Jinzhou Medical University

Kai Gao Jinzhou Medical University

Xiaochun Xu

Sun Yat-Sen University

\section{Ziji Zhang}

Sun Yat-Sen University Zhongshan Ophthalmic Center

\section{Xifan Mei}

Jinzhou Medical University

\section{Research Article}

Keywords: Osteoarthritis, Biomimetic nanoparticles, CD90+ MCSs-derived micro-vesicle, Cartilage regeneration, Anti-inflammatory

Posted Date: January 6th, 2022

DOI: https://doi.org/10.21203/rs.3.rs-1210511/v1

License: (c) (i) This work is licensed under a Creative Commons Attribution 4.0 International License. Read Full License 
Version of Record: A version of this preprint was published at Journal of Nanobiotechnology on March 19th, 2022. See the published version at https://doi.org/10.1186/s12951-022-01367-z. 


\section{Abstract}

Background: Osteoarthritis $(\mathrm{OA})$ is a highly prevalent human degenerative joint disorder that has long plagued patients. Glucocorticoid injection into the intra-articular (IA) cavity provides potential short-term analgesia and anti-inflammation, but long-term IA causes loss of cartilage content. Synovial mesenchymal stem cells (MSCs) reportedly promote cartilage proliferation and increase cartilage content.

Methods: The CD90+ MCSs-derived micro-vesicle (CD90@MV)-coated nanoparticle (CD90@NP) was developed. $\mathrm{CD} 90^{+} \mathrm{MCSs}$ were extracted from human synovial tissue. Cytochalasin $\mathrm{B}(\mathrm{CB})$ relaxed the interaction between the cytoskeleton and the cell membranes of CD90 ${ }^{+} \mathrm{MCSs}$, stimulating CD90@MV secretion. The poly (lactic-co-glycolic acid) (PLGA) nanoparticle was coated with CD90@MV, and a model glucocorticoid, triamcinolone acetonide (TA), was encapsulated in CD90@NP (T-CD90@NP).

Results: CD90@MV membrane proteins were similar to CD90 $\mathrm{MCSs}$, indicating that the CD90@MV bioactivity is similar to the cartilage proliferation-inducing CD90 MCSs. The CD90@NP binding to injury cartilage primary cells was significantly stronger than the erythrocyte membrane-coated nanoparticles (RNP). In the rabbit OA model, long-term IA of T-CD90@NP showed significantly enhanced repair of damaged cartilage than TA and $\mathrm{CD} 90^{+}$MCSs treatments. In the rat OA model, short-term IA of TCD90@NP showed effective anti-inflammatory ability similar to TA treatment. Moreover, long-term IA of TCD90@NP induced cartilage to restart the cell cycle and reduced cartilage apoptosis. T-CD90@NP promotes regeneration of chondrocytes, reduces apoptosis via the FOXO pathway, and influences type 2 macrophage polarization to regulate inflammation through IL-10.

Conclusion: This study confirms that T-CD90@NP promotes chondrocyte proliferation and antiinflammation, improving the clinical glucocorticoid treatment plan.

\section{Highlights}

- Long-term injection of glucocorticoids in the knee joint cavity promotes loss of cartilage content

- CD90-positive stem cell vesicles encapsulated with Triamcinolone acetonide -loaded nanoparticles have good materials

- T-CD90@NPs drive anti-inflammatory properties and promote cartilage regeneration after osteoarthritis

- T-CD90@NPs regulate the polarization of type 2 macrophages to resist inflammation

- T-CD90@NPs promote chondrocyte regeneration through the FOXO signaling pathway

\section{Introduction}

Osteoarthritis (OA) is a highly prevalent human degenerative joint disorder that causes pain and dysfunction, seriously endangering physical and mental health. Worldwide, OA affects 250 million 
people[1]. The disease causes cartilage injury, osteophyte formation, vascular invasion of the articular surface, and synovial inflammation. The confirmed OA risk factors include obesity, aging, sex, previous joint injury, genetics, or anatomical factors, but the exact OA pathogenesis is unclear[2, 3]. Pathological mechanisms of OA include the increase of matrix-degrading enzymes, for example, MMP13, ECM degradation, the reduction of SOX9-related transcription factors, and chondrocyte apoptosis[4]. Therefore, current $O A$ clinical treatment mainly involves injecting drugs into the intra-articular (IA) cavity and surgery. Unfortunately, these treatment strategies are ineffective. Optimized, effective treatments are urgently required.

Early OA clinical treatments involve injecting glucocorticoids and sodium hyaluronate into the joint cavity $[5,6]$. Short-term use of glucocorticoids is beneficial for anti-inflammatory and pain relief. However, long-term use of glucocorticoids may cause chondrocyte cycle arrest and cartilage loss[7-11]. Therefore, the anti-inflammatory and analgesic ability of glucocorticoids for $\mathrm{OA}$ treatment remains limited unless the problem of chondrocyte cycle arrest is solved. Another OA treatment solution to the glucocorticoid limitation involves employing MSCs, which induce proliferation, differentiate into the cartilage, and transport drugs[12,13]. MSCs promote cartilage proliferation and restart the cartilage cell cycle in OA treatment, while the anti-inflammatory and analgesic ability of MSCs is weak[14].

Nanoparticle-based biomimetic DDSs are another OA treatment strategy with fewer side effects[15]. The combination of the natural cell membrane and synthetic NPs disguise NPs as endogenous cells, reducing their elimination and prolonging the NPs therapeutic effects[16]. In this system, NPs are loaded with drugs, and the encapsulated biological cell membrane exerts the cellular biological effects[17, 18]. The most important factor of the outer cell membrane is preparing biological cell membranes to wrap NPs[19]. This NP-based DDS allows glucocorticoids to retain their anti-inflammatory and analgesic effects without affecting the proliferation of cartilage cells.

We used the synovial-derived MSCs to encapsulate glucocorticoid-carrying NPs. However, not all synovium-derived-MSCs promote cartilage proliferation[20, 21]. Reports demonstrated that the human synovial CD90-positive MSCs (CD90 MSCs) significantly reduced after OA and that $\mathrm{CD}^{+} 0^{+} \mathrm{MSC}$ may be involved in cartilage repair after OA[22-24]. Similarly, micro-vesicles extracted from CD $90^{+} \mathrm{MSCs}$ $\left(\mathrm{CD} 90^{+} @ M V\right)$ can promoted cartilage repair[25]. Unlike the traditional membrane extraction method, CD90+@MV benefits from density gradient centrifugation and ultracentrifugation[26, 27]. The cell membrane CD90+@MV extracted from this work is rich in CD90 proteins. Membrane proteins are important in bio-functions.

In this study, CD90+@MV was prepared to wrap NPs loaded with TA, a common clinical glucocorticoid for knee joint injection in OA. The CD90 @MV-coated NPs with TA (T-CD90+@NP) were developed with higher TA than bar NP capacity. CD90 @NP uptake by cartilage cells is better than red blood cell membranecoated NPs (RNP). This study determined the therapeutic efficacy of T-CD90+@NP in rabbit OA treatment using micro-CT and pathological staining analysis. The anti-inflammatory ability of T-CD $90^{+} @ N P$ was 
tested in the early OA stage, and the ability to promote the proliferation of chondrocytes during the late $O A$ stage in the rat model. Finally, the anti-inflammatory and proliferation mechanisms were determined using transcriptomic (RNA-seq)-analysis of a rat model. This study synthesized T-CD90+@NP for the first time and proved the T-CD90 @NP ability to induce the TA-caused chondrocyte cycle arrest while retaining the anti-inflammatory effect of TA. This approach may optimize traditional glucocorticoid injection for treating joint cavities in $\mathrm{OA}$.

\section{Results}

\section{TA has limited long-term efficacy and causes chondrocyte cell cycle arrest in human OA}

The knee cartilage of OA patients with IA and had received 3-4 TA treatments for over one year before testing was used to observe the long-term efficacy of TA in human OA patients. Histomorphological staining objectively proved the condition of tissue repair because the articular cartilage layer is defective after $\mathrm{OA}$. As the disease progresses, the defect is filled with new cartilage or fibrous connective tissue. The H\&E and Masson staining (Fig. 1B) showed that the surface fibrillation area was larger, and the chondrocytes were abnormally distributed in the OA and TA than the normal group.

The Safranin O Fast Green Staining showed that the cartilage polysaccharide level reduced, and the cartilage matrix was severely degraded in the OA and TA groups than the normal group. However, there was no substantial difference in cartilage damage between the OA and TA groups. Quantitative analysis using the OARSI scoring revealed high scores, which indicates poor tissue repair. The results showed that the $\mathrm{OA}$ and TA groups had significantly higher scores than the normal group, but the scores between $\mathrm{OA}$ and TA groups were not substantially different (Fig. 1C).

The SOX9 and MMP13 $\mathrm{IH}$ staining determined the proliferation, differentiation, and degradation of chondrocytes. The results showed that SOX9 expression in the cartilage layer significantly decreased, but MMP13 increased in the OA and TA than the normal group. However, both SOX9 and MMP13 expressions were not substantially different between the OA and TA groups (Fig. 1D, E). Cell cycle-related factors P21, P16 (negative cell cycle regulator), and ki67 (cell proliferation and regeneration marker) were detected to observe chondrocyte cell cycle arrest after TA treatment. The results showed that P21 and P16 expression significantly increased in the cartilage layer, but Ki67 considerably decreased in the OA and TA than the normal group. There was no significant difference in the expression of P21, P16, and Ki67 between the $\mathrm{OA}$ and TA groups (Fig. 1F-H). Altogether, these results revealed that in the long-term, TA treatment causes cartilage tissue structure disorder and chondrocyte cell cycle arrest in OA patients.

\section{Extraction and activity identification of CD90-positive stem cells from human synovium}

The human synovium IF staining results showed that the CD90 expression significantly increased in the normal than the OA group, whereas the expression decreased in the TA group (Fig. 2B). This observation shows that TA treatment continually reduced the number of CD90-positive cells in the synovium after OA. Thus, we infer that $\mathrm{CD} 90^{+} \mathrm{MSC}$ from the synovium are related to TA-induced chondrocyte cell cycle 
arrest after OA. We extracted CD45 (FITC) negative and CD90 (PC7) positive cells from healthy human synovium via fluorescence-activated cell sorting based on adherent growth on the plate and spindle morphology (Fig. 2C). Next, the proliferation ability of the obtained cells (normal CD $90^{+} \mathrm{MSCs}$ ) was tested through different generations (P5, P10, and P15). The DAPI stain results showed that the cells increased from $\sim 300$ to $\sim 2500$ within 72 hours, but the cell proliferation ability was indifferent between generations (Fig. 2D). In addition, normal CD90+ MSCs expressed CD44 (93.2\%) and CD106 (99.3\%), representing common MSCs surface markers (Fig. 2E). Inducing normal CD90 ${ }^{+}$MSCs in osteogenic, adipogenic, and chondrogenic conditioned media verified their differentiation ability. The results showed that Alizarin Red, oil-red-O, or Toluidine blue positively stained the differentiated normal CD $90^{+} \mathrm{MSCs}$ (Fig. $2 \mathrm{~F}$ ). Altogether, these results infer that $\mathrm{CD} 90^{+} \mathrm{MSC}$ from the healthy human synovium have good proliferation and differentiation ability.

\section{Characterization of CD90@NP and its uptake ability by cartilage}

The TEM was used to observe the structure of CD90@NP, which consists of CD90@MV-wrapped NP, prepared for a DDS. The TEM results showed that CD90@NP had a significantly clear core and shell structure, indicating the biofilm coating on the NP surface (Fig. 3B). The CD90@NP size was approximately $154.3 \pm 7.5 \mathrm{~nm}$ (Fig. 3C), slightly larger than NP (103.3 $\pm 6.7 \mathrm{~nm})$. We tested the Zeta potential (Z) of CD90@NP. Zeta potential is the potential of the shear surface, an important indicator of biofilm stability. The results showed that the CD90@NP $\zeta$ potential (approximately $-33.1 \pm 1.6 \mathrm{mV}$ ) was similar to CD90@MV (natural cell membrane, approximately -52.67 $\pm 1.5 \mathrm{mV}$ ). The CD90@NP was stable within one week with a little size change (Fig. 3D). CD90@MV has good biocompatibility because it is derived from $\mathrm{CD} 90^{+} \mathrm{MSC}$ in the synovium. However, NP has poor biocompatibility and causes blood clotting. Thus, the CD90NP biocompatibility was tested against CD90MV for investigating the CD90@NP stability in the blood to reduce the NP-caused blood clotting. Briefly, CD90@NP and NP were incubated with FBS, followed by detection of CD90@NP and NP coagulation. Coagulation was detected by testing the turbidity change over time. The coagulation results showed that NP had higher opacity $(560 \mathrm{~nm})$ within 20 minutes compared with CD90@NP, while the CD90@NP group remained stable within 120 hours (Fig. 3E).The good biocompatibility and stability of CD90@NP may be related to CD90@MV being wrapped on the surface of CD90NP, acting as a shield.

Triamcinolone acetonide-loaded CD90@MV (T-CD90@NP) was prepared as the following description. In brief, The T-CD90@NP preparation was optimized by inputting TA in the PLGA core (10\% w/w). The TA release from T-CD90@NP was tested within $200 \mathrm{~h}$ to determine the TA capacity of CD90@NP. The TA release rate in T-CD90@NP decreased compared with T-NP, which might be related to the CD90@MV cover on the surface of T-CD90@NP (Fig. 3F). The low TA release rate is beneficial for prolonging the TA efficacy for joint cavity treatment.

The protein maintained on the CD90@NP surface (from CD90@MV) is essential for its biological function, such as cartilage repair. Thus, the CD90 MSCs, the CD90@MV, and the CD90@NP protein composition were tested using SDS-PAGE. The results of SDS-PAGE showed that CD90 ${ }^{+} \mathrm{MSCs}$, the 
membrane of CD90+ MSCs, CD90@MV, and CD90@NP have similar protein compositions (both 80Da and $22 \mathrm{kDa}$ have similar bands). Thus, the important protein bands (in the red-dotted frame) such as CD90 (22 kDa) and CD44 (80 kDa) were found (Fig. 3G). CD90+ MSCs, the membrane of CD90+ MSCs, CD90@MV, and CD90@NP were subjected to western blotting to identify further the two potential functional proteins, CD90 and CD44. CD90 ${ }^{+}$MSCs, the membrane of CD90+ MSCs, CD90@MV, and CD90@NP maintained similar expressions of CD44 and CD90 (Fig. 3H).

We constructed a physical damage model of chondrocytes (scratch model) and used DID (red) to stain TCD90@NP and T-RNP and verify whether the damaged primary chondrocytes will take up CD90@NP. The damaged primary chondrocytes take up more T-CD90@NP than the T-RNP group, probably because CD90@NP inherits the properties of $\mathrm{CD} 90^{+} \mathrm{MSC}$ s extracellular vesicles (Fig. 3I). Moreover, several assays were conducted to test T-CD90@NP cytotoxicity, including 3-(4,5-Dimethylthiazol-2-yl)-2,5diphenyltetrazolium bromide (MTT) assays in vitro, hepatotoxicity, routine blood tests and HE staining of important organs. The results of MTT assays showed that IL-1 $\beta$ treatment decreased the viability of primary chondrocytes, and the cell activities of T-CD90@NP and CD90@NP were the highest at the concentration of $2 \mathrm{mg} / \mathrm{mL}$ (Fig. 4A, B). The serological ELISA test was used to observe the effect of TCD90@NP on liver function, kidney function and the circulatory system in rats. The results showed no abnormalities in alanine aminotransferase (ALT), aspartate aminotransferase (AST), creatinine (CREA), urea nitrogen (BUN), white blood cell (WBC), red blood cell (RBC), hemoglobin (HGB) and platelet (PLT) levels (Fig. 4B-I). Similarly, the HE staining of the heart, liver, spleen, lung and kidney in all groups were normal (Fig. 4J).

\section{T-CD90@NP promote articular cartilage repair in OA model rabbit}

The study employed micro-CT to observe the formation of osteophytes in the knee joint cavity and subchondral bone defects after OA. The micro-CT results showed that 6 months after treatment, the subchondral bone surface in the T-CD90@NP group was smooth and similar to the normal group (Fig. 5B). However, the subchondral bone surface in the other groups was rough and displayed varying degrees of bone defect. Meanwhile, the subchondral bone in the OA and T-NP groups increasingly collapsed.

Moreover, severe damage induced numerous osteophytes, and numerous osteophytes (red cubes marked osteophyte, region of interest, $\mathrm{ROI}$ ) appeared in each group of joint cavities after OA. Similarly, the volume of osteophytes in the T-CD90@NP significantly decreased than in the other treatment groups (Fig. 5C). Likewise, the T-CD90@NP group showed lower osteophyte scores than the other groups (Fig. 5D). Altogether, these findings indicate that at 6 months, T-CD90@NP is beneficial for subchondral bone and osteophyte formation in the OA model rabbit.

The most damaged femoral trochlear cartilage was selected for pathological examination to observe the changes of cartilage structure 6 months after treating the OA model rabbit. The H\&E staining and microCT results were similar. The chondrocytes in the repaired T-CD90@NP tissue were neatly arranged, similar to the normal group. However, the repaired cartilage appeared as disorderly, fibrous, and loose tissue in 
the other treatment groups. The Safranin 0 fast green results showed that the T-CD90@NP and normal groups had the best cartilage repair compared with the other treatment groups. Masson staining is specific for fibrous tissue. The Masson staining results showed that the other treatment groups had increased fibrous tissue, exhibiting a disordered tissue structure than the T-CD90@NP group (Fig. 6A). Moreover, the OARSI scores (histomorphology scores) were consistent with the staining results. The OARSI scores of the T-CD90@NP group were significantly lower than the other treatment groups but higher than the normal group (Fig. 6B).

\section{T-CD90@NP regulates joint cavity inflammation by promoting the macrophage polarization to the M2 phenotype in OA model rats}

Regulating the inflammation of the joint cavity microenvironment after $\mathrm{OA}$ effectively promotes cartilage regeneration, and the synovium macrophages regulate the inflammation levels of the microenvironment. We injected T-CD90@NP into the knee joint cavity in a rat OA model to verify whether the antiinflammatory ability of T-CD90@NP is similar to TA after OA. IF staining for the proinflammatory cytokines IL- 6 and the anti-inflammatory cytokine IL-10 in the joint synovium reflects the inflammation level of the joint cavity microenvironment (Fig. 7A). Two weeks after treatment, the IL-10 expression in TCD90@NP significantly increased, similar to the TA group. However, the expression of IL-6 decreased compared with the other groups, a trend similar to the TA group. These results indicate that T-CD90@NP and TA have similar anti-inflammatory abilities. Two weeks after OA, we performed IF staining for three macrophage polarization markers (CD68, CD206, and iNOS) to explore further the T-CD90@NP mechanism of regulating inflammation. Both IL-6 and IL-10 induce the polarization of macrophages (Fig. 7B). CD68 and iNOS significantly decreased in the T-CD90@NP and TA groups, while the expression of CD206 increased in the T-CD90@NP and TA groups than the other treatment groups (Fig. 7C). These results indicated that after 2 weeks of treatment, T-CD90@NP induces synovium macrophages to polarize to $\mathrm{M} 2$ phenotype in OA model rats.

\section{T-CD90@NP promotes cartilage regeneration and reduce cartilage apoptosis in OA model rats}

Cartilage damage and apoptosis caused by OA are key yet difficult points of treatment. Therefore, methods to reduce cartilage apoptosis and promote cartilage regeneration need attention. In a rat OA model, we injected T-CD90@NP into the knee joint cavity to detect cartilage regeneration and apoptosis after T-CD90@NP treatment. Six months after OA, we observed cartilage apoptosis and cell cycle by IF and qPCR. The cell regeneration marker (EDU) was enhanced in the cartilage layer of T-CD90@NP and CD90@MV than the other treatment groups. There was little difference in EDU expression between TCD90@NP and CD90@MV groups (Fig. 8A, B).

However, the cell apoptosis marker (TUNEL) expression decreased in the T-CD90@NP than in the other treatment groups. There was a decrease in T-CD90@NP compared with CD90@MV, probably due to the early anti-inflammatory ability of the enclosed TA (Fig. 8C). The qPCR detected key cell cycle factors to explore the impact of T-CD90@NP on the chondrocyte cycle. The cell cycle promoter (Cyclin and CDK family) significantly increased in the T-CD90@NP and CD90@MV groups than the other groups. However, 
the cell cycle inhibitor (CDKN family) decreased in the T-CD90@NP than the other treatment groups, similar to the CD90@MV group (Fig. 8D). The ability of T-CD90@NP to promote cartilage cell regeneration may be related to the $\mathrm{CD} 90 @ \mathrm{MV}$ surface cover because synovial mesenchymal stem cells produce CD90@MV, which promotes cartilage proliferation.

Next, we used mRNA sequencing and bioinformatic analysis to explore the T-CD90@NP molecular mechanism promoting cartilage proliferation and anti-inflammation in rats 6 months after OA. TCD90@NP substantially changed the expression of many genes in the cartilage of rats 6 months after OA (Fig. 9A-B). The KEGG pathway analysis showed that the top 100 high-abundant mRNAs enriched cellular processes, environmental information (Fig. 9C). The study focused on the genes for cell growth and death, considering the T-CD90@NP biological test results. Indeed, the FOXO signaling pathway was enriched in the T-CD90@NP cartilage after OA (Fig. S1). Both qPCR mRNA results showed that the IL-10, IGF1, cyclin B, PLK, and catalase expression increased, while the IRS, SGK, and FOXO1 decreased in the TCD90@NP than the OA group (Fig. 9D). The other KEGG enriched pathways include the JAK-STAT, insulin, PI3K-Akt, and FOXO signaling pathways. In summary, these enriched pathways possibly regulate cartilage regeneration (Fig. 9E).

\section{Materials And Methods}

\section{Human cartilage and synovium}

The human OA cartilage and synovium (OA group) were obtained from patients undergoing total knee replacement surgery ( $n=5$; aged $61.00 \pm 3.71$ years; one male and four females). The TA group includes the human $\mathrm{OA}$ cartilage and synovium group that had received TA injections in the joint cavity. These samples were obtained from patients undergoing total knee replacement surgery $(n=5$; aged $56.00 \pm$ 4.21 years; two males and three females; received 3-4 TA intra-articular injections). Normal control cartilage and synovium (normal group) were obtained from patients who had suffered traffic accidents, with no history of the arthritic disease ( $n=4$; aged $34.17 \pm 7.32$ years; two males and two females). The human cartilage was stained with HE, Masson, Safranin O, Immunohistochemistry (P21, P16, MMP13, and SOX9), and immunofluorescence (Ki67/DAPI). The human synovium was stained with immunofluorescence (CD90/DAPI). Patients provided written consent, and the Ethics Committee of the First Affiliated Hospital of Jinzhou Medical University (Jinzhou, China) approved the study before the human tissue samples were harvested.

\section{Isolation and culture of $\mathrm{CD}^{+} 0^{+} \mathrm{MSCs}$}

Fresh knee synovium was obtained from humans (who had suffered traffic accidents with no history of arthritic disease). The synovium was cut into pieces of $1 \mathrm{~mm}^{3}$ under aseptic conditions and digested in 4 $\mathrm{mg} / \mathrm{ml}$ type I collagenase (prepared in DMEM). After 120 minutes of digestion at $37^{\circ} \mathrm{C}$, the synovium was filtered and centrifuged to obtain a synovial single-cell suspension. A culture medium containing $10 \%$ FBS (prepared in DMEM) was added to the culture flask and cultured at $37^{\circ} \mathrm{C}$. 


\section{Flow cytometry}

Single cells were resuspended and incubated with flow cytometry antibodies (CD90/CD45) at $4^{\circ} \mathrm{C}$ for 30 minutes. CD90+CD45- cells were sorted using the BD Influx cell sorter (BD Biosciences, NJ, USA). The isolated cells were cultured with DMEM/F12 (Gibco, TX, USA) supplemented with $10 \%$ fetal bovine serum (Gibco, TX, USA) and $100 \mathrm{IU} / \mathrm{ml}$ penicillin/streptomycin (Invitrogen, MA, USA) at $37^{\circ} \mathrm{C}$ in a $5 \% \mathrm{CO}_{2}$ atmosphere. Cells were passaged when they reached $90 \%$ density.

Cells at passage five were used to evaluate stem cell surface markers by flow cytometry. All cultured cells were digested into single cells with trypsin and resuspended in PBS with appropriate antibodies (CD44/CD106). After incubation at $4^{\circ} \mathrm{C}$ for 30 minutes, cells were washed thrice using PBS and tested using influx flow cytometers (BD Biosciences, NJ, USA).

The flow antibodies used in this study were: CD90-PECY7 (dilution 1:200), CD45-FITC (dilution 1:200), CD44-PE (dilution 1:200), and CD106-APC (dilution 1:200) (BD Biosciences, NJ, USA).

\section{Viable cell count}

The Celllnsight HCS and HCA systems (Thermo Fisher Scientific, MA, USA) detected changes in the number of nuclei through nuclear staining (DAPI immunofluorescence stain) of CD90 ${ }^{+}$MSCs cells at the fifth (P5), tenth (P10), and fifteenth (P15) generations of the culture.

\section{CD $90^{+}$MSCs differentiation ability}

The adipogenic (HUXUC-90031), osteogenic (HUXUC-90021), and chondrogenic (HUXUC-90041) induction media (Cyagen Biosciences, CA, USA) were used to evaluate the ability of $\mathrm{CD} 90^{+} \mathrm{MSC}$ s to differentiate into osteocytes, adipocytes, and chondrocytes. After 21 days, Alcian blue, Alizarin red, and Oil red 0 staining detected the differentiation ability of $\mathrm{CD} 90^{+} \mathrm{MSCs}$.

\section{Immunofluorescence and immunohistochemical staining}

The cartilage of human and rabbit knee joints was decalcified using EDTA. The softened cartilage tissue and fresh synovium were washed with low-temperature PBS $\left(4^{\circ} \mathrm{C}\right)$, embedded in paraffin, and sectioned $(2 \mu \mathrm{m})$. After deparaffinization and antigen retrieval, the obtained paraffin sections were blocked with $10 \%$ goat serum for 2 hours at room temperature. For IF staining, the following antibodies were used to detect antibodies: anti-Ki67 (ab238020, 1:800), anti-Phalloidin (8878, 1:20), anti-DAPI (ab104139, 1:1), anti-IL-10 (ab9969, 1:1000), anti-IL-6 (ab259341, 1:1000), anti-CD68 (ab125212, 1:500), anti-CD206 (ab64693, 1:1000), and anti-iNOS (ab178945, 1:800) from Abcam (Cambridge, UK), anti-CD90 (sc-53116, 1:1000) Santa Cruz biotechnology Inc (TX, USA), EDU (5-ethynyl-2'-deoxyuridine, E10187, $1 \mathrm{mg}$ for intra-articular injection, once a week) (Thermo Fisher Scientific, MA, USA), and TUNEL stain (11684817910, 1:300) (Roche, Basel, Switzerland).

For IH staining, the following antibodies were used to detect antibodies: anti-SOX9 (EPR14335, 1:500), anti-MMP13 (EPR21778, 1:1000), anti-P21 (HUG0291, 1:500), anti-P16 (ab151303, 1:500), and Fontana- 
Masson stain (ab150669) from Abcam (Cambridge, UK), Safranin O Fast Green Stain, and HE stain from Servicebio (Wuhan, China). Photographic tests were taken after incubation with appropriate secondary antibodies using the LCM800 microscope (Zeiss, Jena, Germany).

\section{Animal experiment}

This Institutional Animal Care of Jinzhou Medical University approved the study protocol. Thirty-five New Zealand white rabbits (6-months old males) and 42 adult SD rats (6-months old males) were purchased from and housed at the experimental animal center of Jinzhou medical university. The rabbits were randomly divided into seven groups of five rabbits each. The groups included normal (the skin was cut without damaging the ligaments), OA (rabbits underwent anterior cruciate ligament transection (ACLT) surgery to injure the joint, and the OA model was successfully induced after 2 weeks), TA (the OA model + $12 \mathrm{mg}$ TA, IA once a week for 24 weeks), T-NP (OA model + NP wrapped TA, 12mg IA once a week for 24 weeks), T-RNP (OA model + RMV wrapped TA, 12mg once a week for 24 weeks), CD90@MV (OA model + CD90@MV, 12mg IA once a week for 24 weeks), and T-CD90@NP (OA model + T-CD90@NP, 12mg IA once a week for 24 weeks), respectively.

Subsequently, the rats were randomly divided into seven groups, with six randomly assigned rats per group. The groups included: normal (the skin was cut without damaging the ligaments), OA (rats underwent anterior cruciate ligament transection (ACLT) surgery to injure the joint, and the OA model was successfully induced after 2 weeks), TA (OA model $+0.25 \mathrm{mg}$ TA, IA once a week), T-NP group (OA model + NP wrapped TA, $0.25 \mathrm{mg}$ IA once a week), T-RNP (OA model + RMV wrapped TA, 0.25mg IA once a week), CD90@MV (OA model + CD90@MV, 0.25mg IA once a week), and T-CD90@NP (OA model + TCD90@NP, 0.25mg IA once a week), respectively. After 2 weeks of treatment, three rats in each group were randomly selected for synovial tests, and the other three were taken for knee joints tests. After the OA model, the rabbits and rats were allowed to move and eat freely.

\section{Preparation of CD90@MV, CD90@NP, T-NP, T-RNP, and T- CD90@NP}

CD90@MV: CD90MSCs were taken from human synovium (normal group) by fluorescence-activated cell sorting and cultured in DMED supplemented with 10\% FBS. The CD $90^{+} \mathrm{MSC}$ were washed thrice with PBS and subsequently incubated in $5 \mathrm{~mL}$ FBS-free DMEM with $10 \mu \mathrm{g} / \mathrm{mL}$ CB (Abcam, Cambridge, U.K.) for 2 hours at $37^{\circ} \mathrm{C}$ to get CD90@MV. The CD90@MV and cells were detached from the cell culture flask using $5 \mathrm{~mL}$ DMEM and vortexed for 8 minutes at $37^{\circ} \mathrm{C}$ to isolate CD90@MV. Subsequently, $5 \mathrm{~mL}$ FBS was added to the tube to obtain a final concentration of $50 \%$ FBS. The mixture was centrifuged at $3000 \mathrm{rpm}$ for 5 minutes to remove cells, impurities, and large CD90@MV aggregates. Next, the obtained supernatant was centrifuged at 8000 rpm for 10 minutes to get the CD90@MV. The obtained CD90@MV was washed thrice with $0.25 \%$ EDTA (diluted using PBS) to remove the nucleus and cytoplasm. The CD90@MV protein quantity was determined using a BCA assay (Thermo Fisher Scientific, MA, USA). 
CD90@NP: The CD90@MV membrane-coated NP (CD90@NP) was prepared following a previous study[28]. Briefly, 0.7 dL/g carboxy-terminated 50:50 PLGA (LACTEL Absorbable Polymers, Birmingham, UK) was used to form the PLGA NP cores using a nanoprecipitation method. The PLGA was dissolved in acetone solution $(10 \mathrm{mg} / \mathrm{mL})$, and $1 \mathrm{~mL}$ of the PLGA solution was added to $2 \mathrm{~mL}$ deionized water to remove the acetone solution via the vacuum method. The resulting NP solution was mixed with CD90@MV at a ratio of 1:10 (w/w, protein: PLGA) and sonicated using a water bath sonicator (FPMRCDCS-250H, Fuguang, China) for 5 minutes at $80 \mathrm{~W}$.

NP, T-RNP, and T-CD90@NP: Red blood cell membranes were collected from healthy human and coated with nanoparticles (RNP) following previous methods[28]. The RNP and CD90@NP were labeled with DiD $(5 \mu \mathrm{M}$, Fanbo Biochemicals Co. Ltd, Beijing, China) at the concentration of $0.2 \%(w / w)$ for 10 minutes. Triamcinolone acetonide (18026, Cayman Chemical Company, MI, USA)-loaded NP (T-NP), RNP (T-RNP), and CD90@NP (T-CD90@NP) were prepared by adding 20\% (w/w) TA to respective solutions during the PLGA core preparation.

The structure micro humid of the CD90@NP was observed by the HT7800 transmission electron microscope (Hitachi Ltd, Tokyo, Japan). The $\zeta$ potential (mA) and particle size (nm) of the CD90@MV, NP, RNP, and CD90@NP were detected by a Malvern Zetasizer Nano ZS90 nanoparticle size system (Malvern, UK). The T-CD90@NP was collected via centrifugation at 12 000 for 45 minutes and dissolved in dimethyl sulfoxide to determine the DLC and EE of TA. The TA content in T-CD90@NP was tested by ELISA (Abnova, Taipei, Taiwan). The following formula calculated the DLC and EE:

$\operatorname{DCL}(\%)=\frac{\text { TAencapsulatedinT }- \text { CD90 @ NP }}{\text { weightofT }- \text { CD90 @ NP }} \times 100 \%$

$\mathrm{EE}(\%)=\frac{\text { TAencapsulatedinT }-\mathrm{CD} 90 @ \mathrm{NP}}{\text { totalTA }} \times 100 \%$

\section{Preparation and Characterization of CD90@NP}

The CD90@NP size change was detected for 7 days at $4^{\circ} \mathrm{C}$, to evaluate the stability of CD90@NP. The CD90@NP was incubated with 50\% FBS (500-S, AusgeneX, Queensland, Australia), and the turbidity over time was detected using a microplate reader at $560 \mathrm{~nm}$ to evaluate the CD@90NP stability in blood[29]. To the rate of TA release from T-CD90@NP was determined by adding $2 \mathrm{mg}$ of T-CD90@NP to $1 \mathrm{~mL}$ of $0.5 \%(\mathrm{v} / \mathrm{v})$ Tween 80 solution (diluted by DMEM) in dialysis bags, then centrifuging after 10, 20, 30, 40, $50,60,70,90,100$, and 120 hours $(n=3)$ to obtain the $1 \mathrm{~mL}$ of supernatant. After that, the amount of TA was measured by ELISA.

\section{The CD90@NP Protein Composition}

The CD90@NP protein composition was investigated by SDS-PAGE. Briefly, extracted proteins from the synovial mesenchymal stem cells, CD90 MSCs membrane, CD90@MV and CD90@NP, were quantified to $2 \mathrm{mg}$ by BCA protein concentration detection. After electrophoresis and transfer, the protein was stained 
using Coomassie Brilliant Blue and imaged. Similarly, anti-CD90 (ab225), anti-CD44 (ab9524) and Na/K ATPase (ab167390) from Abcam (Cambridge, UK) were stain and imaged.

\section{In vitro binding of CD90@NP}

We conducted an in vitro phagocytosis experiment of CD90@NP by primary chondrocytes to determine whether chondrocytes can phagocytose CD90@NP. Briefly, the extracted primary chondrocytes from suckling rabbits were inoculated on 24-well plates. The IL-1 $\beta(10 \mu \mathrm{g} / \mathrm{L}$, Peprotech, USA) was used for cell damage (treated $24 \mathrm{~h}$ at $37^{\circ} \mathrm{C}$ ). Next, the DiD-labeled CD90@NP was added to the chondrocytes culture for 8 hours at $4{ }^{\circ} \mathrm{C}$ and washed thrice with HBSS (Gibco, TX, USA). The DiD-labeled RNP was the control group. After that, the absorbance at $633 \mathrm{~nm}$ (DiD) and $488 \mathrm{~nm}$ (phalloidin, labeled chondrocytes) were imaged by an LCM800 microscope.

\section{Cell Viability Assays}

Primary chondrocytes were cultivated in Dulbecco's Modified Eagle Medium (DMEM, Gibco, USA) and $10 \%$ fetal bovine serum (FBS, Gibco, USA) after which MTT assays were conducted to evaluate cell viability. In brief, primary chondrocytes were treated with CD90@NP or T-CD90@NP $(0,0.5,2,5,10$ and 50 $\mathrm{mg} / \mathrm{mL})$, then treated with IL- $1 \beta$ for $24 \mathrm{~h}$ at $37^{\circ} \mathrm{C}$. Subsequently, MTT ( $20 \mu \mathrm{M}$, Sigma- Aldrich, USA) was added to each well and plates were incubated for $4 \mathrm{~h}$ at $37^{\circ} \mathrm{C}$. Dimethyl sulfoxide (150 $\mu \mathrm{l}$, Sigma- Aldrich, USA) was added to each well and the absorbance at $490 \mathrm{~nm}$ was recorded.

\section{ELISA detection}

After 10 minutes of natural coagulation at room temperature, the blood $(0.3 \mathrm{~mL})$ from rats was centrifuged for 20 minutes (3000 rpm), and the supernatant was collected for subsequent analyses. Next, the alanine transaminase (ALT), aspartate transaminase (AST), creatinine (CREA), blood urea nitrogen $(B U N)$, white blood cell (WBC), red blood cell (RBC), hemoglobin (HGB) and platelet (PLT) levels were detected by ELISA (GM1149, Servicebio, China), then the absorbance at $340 \mathrm{~nm}$ was recorded.

\section{Micro-CT detection}

The knee joint of rabbits from the different groups was examined using a micro-CT imaging system (Siemens, Munich, Germany). The images were further reconstructed and analyzed using the Siemens Multimodal 3D Visualization software (Siemens, Munich, Germany) to calculate the region of interest (ROI, red cubes marked osteophyte) volume.

\section{qPCR and RNA-Sequence Detection}

RNA isolation and the qPCR analysis were performed as described. Briefly, the total RNA was extracted from cartilage tissues using the TRIzol reagent (Invitrogen, MA, USA). RNA (1 mg) was reverse transcribed using the RevertAid First-Strand cDNA Synthesis Kit (Thermo Fisher Scientific, MA, USA) to obtain cDNA. The cDNA templates were analyzed using qPCR with the SYBR Green reagent (Roche, Basel, Switzerland) and pre-designed primers (Sangon Biotech, Shanghai, China). 
The $\mathrm{Ct}$ values were normalized using GAPDH. The relative mRNA abundance was calculated using the $\triangle \mathrm{Ct}$ method [relative mRNA abundance $=2-(\mathrm{Ct}$ gene of interest $-\mathrm{Ct}$ GAPDH)]. The qPCR experiments were performed in triplicate. The rat primer sequences are listed in Table 1. For RNA-seq experiments, isolated RNA samples were sent to Huada Gene Research Institute (Guangdong, China) for sequencing. The raw tag data were produced using the sequencing-by-synthesis method, and the institute processed the transcriptomic data.

Table 1

qPCR primers for key factors of rat chondrocyte cycle.

\begin{tabular}{|llll|}
\hline Name & Gene Name & Forward (5'>3') & Reverse (5'>3') \\
\hline Gapdh & GAPDH & GCATCTTCTTGTGCAGTGCC & GATGGTGATGGGTTTCCCGT \\
\hline Cyclin D1 & CCND2 & ACCTGTGAGGAAGCCATTCG & CCAGCGTGTCCCTTCTCATT \\
\hline Cyclin D3 & CCND3 & ACACGCGTCGCTTCTCCTA & TGTGACATCTGTGGGAGTGC \\
\hline CDK4 & CDK4 & ACCAGGATCTCCCACTAGCA & TCAGGTCCCGGTGAACAATG \\
\hline CDK6 & CDK6 & GGCCGCAGTAGTCAGTTACC & CCAACACTCCAGAGGTCCAC \\
\hline Cyclin E2 & CCNE2 & CGCAGTAGCCGTTTACAAGC & TCACTGCAAGCACCATCAGT \\
\hline Cyclin A2 & CCNA2 & GTCAACCCCGAAAAAGTGC & GCCTTCCATGTGTCTGACCAA \\
\hline CDK2 & CDK2 & AGGCGGCAACATTGTTTCAA & GACAGGGACTCCAAAGGCTC \\
\hline Cyclin B1 & CCNB1 & GGTCGATGTGGAGCAGCATA & GGCAAAATGCACCATGTCGT \\
\hline Cyclin B2 & CCNB2 & TGGCTGGTCCAAGTCCATTC & TGTGCTGCATGACTTCCAGT \\
\hline CDK1 & CDK1 & AGGACCAGCTCACAAAAGGG & GTGGAAAAGCGGCTTCTTGG \\
\hline P16 & CDKN2A & AACACTTTCGGTCGTACCCC & CTCCCTCCCTCTGCTAACCT \\
\hline P21 & CDKN1A & AAGCCCGAGTTCCTGCTAAC & ATCGGCGCTTGGAGTGATAG \\
\hline P27 & CDKN1B & GACTCACTCGCGGCTCC & TGTTTACGTCTGGCGTCGAA \\
\hline
\end{tabular}

\section{Statistical Analysis}

The data were analyzed using GraphPad Prism 8 (GraphPad Software Inc., CA, USA). Significant differences were evaluated using an unpaired Student's t-test for comparing the two groups and one-way ANOVA for multiple-group comparisons. The data are expressed as the mean \pm standard deviation (SD), and a $P$-value $<0.05$ indicated a significant difference.

\section{Discussion}

The glucocorticoid IA promotes analgesia and reduces inflammation after OA[30]. However, its long-term use can lead to loss of cartilage content[31]. It is thus important to solve the problem of the loss of 
cartilage content but retain its anti-inflammatory and analgesic ability. Notably, injection of synovial mesenchymal stem cells can effectively increase the chondrocytes content and promote cartilage regeneration after $\mathrm{OA}[32]$. Proposals to construct complexes of synovial mesenchymal stem cells combined with glucocorticoids to incorporate anti-inflammatory, analgesic, and cartilage content promotion abilities have thus been put forward. Some DDSs, such as PEGylated liposomes/NPs, have been designed based on the excellent drug loading and slow-release ability to address this challenge. Previous studies postulate that NP has a good loading and slow-release capacity for TA.

In this study, long-term IA of TA had limited therapeutic effects on the cartilage of patients after OA. This phenomenon was attributed to the arrest of the chondrocyte cycle, leading to the loss of cartilage content. This challenge was addressed by preparing NPs loaded with TA wrapped with synovial mesenchymal stem cell membranes. CD90 ${ }^{+}$MSCs were extracted from the human synovial tissue, and cytochalasin B was subsequently used to relax the interaction between the membrane and cytoskeleton of $\mathrm{CD} 90^{+} \mathrm{MSC}$ s to stimulate CD90@MV secretion. CD90 $\mathrm{MSC}$ were chosen because they decrease in the synovium of OA patients under TA treatment and the numerous reports highlighting their importance

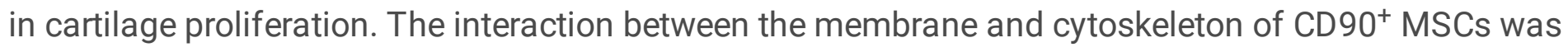
subsequently weakened because the $\mathrm{CD} 0^{+} \mathrm{MSCs}$-associated ability to promote cartilage proliferation and activate cell cycle is significantly mediated by the membrane protein function, and thus cloaking of NP with bio-membranes to maintain the protein activity is mostly necessary. The traditional process of membrane isolation was not used because of its complexity and involves nuclear disposal, cell lysis, and centrifugation, which results in inefficiency and the loss of the membranes protein activity[33, 34]. The NP loaded with TA was then wrapped in CD90@MV to prepare T-CD90@NP. CD90@NP exhibited great stability and ability to release TA. Both CD90@MV and CD90@NP had similar protein compositions and were rich in $\mathrm{CD} 90$, inducing proliferation function, and CD44 proteins, inducing cell adhesion function[35]. These findings suggested that CD90@NP can perform protein functions like CD90 $\mathrm{MSC}$ and CD90@MV. Moreover, CD90@NP can be taken up in large quantities by primary chondrocytes than RNP (bar) after injury. Succinctly, T-CD90@NP has good material and biological properties.

Rabbit OA animal models were used to verify the efficacy of T-CD90@NP, and rat OA animal models were used to explore the molecular mechanisms. TA IA once a week for 24 weeks led to limited cartilage repair in the rabbit model. These findings were similar to those of the OA group. However, T-CD90@NP effectively repaired the cartilage tissue after OA. Recent studies postulate that short-term TA IA effectively reduces the inflammation level in the joint cavity and has a great analgesic effect. In contrast, long-term TA IA has limited repair to the cartilage tissue. The long-term effects of TA are attributed to the loss of cartilage content. TA activates cell cycle inhibitors and inhibits the cell cycle activating factors, leading to cell cycle arrest. The cell cycle arrest deactivates chondrocytes and reduces the secretion of the cartilage matrix, thereby reducing the ability of cartilage tissue to repair[36]. The rat OA model was subsequently used to explore the mechanism of T-CD90@NP against cartilage loss caused by TA. Recent studies postulate that synovial inflammation significantly impacts cartilage repair and is closely related to $O A$ development[37, 38]. M2-type macrophages reduce inflammation in the microenvironment of the synovial 
tissue by secreting IL-10, which induces cartilage repair[39]. Notably, T-CD90@NP reduced synovial inflammation and promoted the polarization of macrophages to the $\mathrm{M} 2$ type in the synovium during the early stages of treatment. Similar findings were observed upon TA treatment. The anti-inflammatory ability of T-CD90@NP is associated with its retention in TA wrapped in NP.

Moreover, long-term treatment using T-CD90@NP effectively reduced chondrocyte apoptosis and increased their proliferation in the damaged area in the rat OA model. T-CD90@NP increased the cell cycle promoting factor (Cyclin and CDK family) but decreased the cell cycle inhibitor (CDKN family). Its ability to restart the cell cycle was attributed to the CD90@MV covered on its surface, which retained the ability of $\mathrm{CD} 90^{+} \mathrm{MSC}$ to promote cartilage proliferation.

The study further explored the molecular mechanisms of T-CD90@NP in promoting chondrocyte proliferation and anti-inflammation. The differentially expressed genes upon T-CD90@NP treatment were found to be enriched in cell growth and death. These findings were based on the pro-proliferation and anti-inflammatory ability of T-CD90@NP. Notably, the differentially expressed genes were enriched in the FOXO signaling pathway, associated with varying biological phenomena, such as cell proliferation, apoptosis, and DNA damage caused by oxidative stress[40, 41]. T-CD90@NP was found to regulate the insulin, PI3K/AKT/FOXO, and Jak/STAT pathways. It can reduce the phosphorylation level of FOXO to increase cyclinB and PLK to restart the cell cycle and catalase to increase DNA repair. It can also increase IL-10, which is secreted by type 2 macrophages, consequently promoting the polarization of type 2 macrophages. Collectively, T-CD90@NP promotes the regeneration of chondrocytes and reduces apoptosis via the FOXO pathway. It also influences the polarization of type 2 macrophages to regulate inflammation by increasing IL-10 secretion.

\section{Conclusion}

This study successfully used NP to combine CD90+ MSCs with TA (T-CD90@NP) to combat the cartilage loss caused by TA after OA. The study demonstrates that CD90@NP is a promising material for mimicking MSCs and can be employed in drug loading. Notably, T-CD90@NP retains the ability to promote cartilage proliferation ( $\mathrm{CD} 90^{+} \mathrm{MSCs}$ ) and anti-inflammation (TA). This system provides a novel strategy for the clinical application of stem cells and glucocorticoids after OA to improve the therapeutic outcomes of patients. It also provides baseline information for future studies related to the subject.

\section{Abbreviations}

Osteoarthritis (OA), intra-articular (IA), mesenchymal stem cells (MSCs), nanoparticle (NP), drug delivery systems (DDSs), CD90+ MCSs-derived microvesicle, CD90+ MCSs)-coated nanoparticle (CD90@NP), cytochalasin B (CB), poly(lactic-co-glycolic acid) (PLGA), Triamcinolone acetonide (TA), Triamcinolone acetonide encapsulated in CD90@NP (T-CD90@NP), erythrocyte membrane-coated nanoparticles (RNP), matrix metalloproteinases (MMP13), extracellular matrix of chondrocytes (ECM), chondrocyte differentiation and proliferation (SOX9), Mesenchymal stem cells (MSCs), nanoparticle (NP), drug delivery 
systems (DDSs), human synovial CD90-positive MSCs (CD90 MSCs), microvesicles extracted from CD90+ MSCs (CD90+@MV), CD90+@MV coated NPs with TA (T-CD90+@NP), drug delivery system (DDS), Osteoarthritis Research Society International (OARSI), Transmission Electron Microscope (TEM), high content screening (HCS), high content analysis (HCA), anterior cruciate ligament transection (ACLT), standard deviation (SD), anti-inflammation (TA), Immunofluorescence (IF), immunohistochemical (IH), bicinchoninic acid protein (BCA), poly (lactic-co-glycolic acid) (PLGA), DID (1,1'-dioctadecyl-3,3,3',3'tetramethylindodicarbocyanine, 4-chlorobenzenesulfonate salt), drug loading capacity (DLC), encapsulation efficiency (EE), Hank's balanced salt solution (HBSS).

\section{Declarations}

\section{Declaration of competing interest}

The authors declare that they have no known competing financial interests or personal relationships that could influence the work reported in this paper.

\section{Acknowledgments}

Sincere thanks to the Department of Orthopedics, the First Affiliated Hospital of Jinzhou Medical University, and the Animal Center of Jinzhou Medical University.

\section{Author contribution}

Yuanlong Li: animal test and biomaterial preparation; Qingqiang Tu: animal test and Micro-CT detection; Dongmei Xie: stem cell extraction, statistical analysis; Shurui Chen: data processing; Kai Gao: clinical data processing; Xiaochun Xu: cell flow cytometry; Ziji Zhang: manuscript writing; Xifan Mei: the overall experimental design.

\section{Funding}

This work was supported by the National Natural Science Foundation of China (Grant No. 82102667, No.81874014) and the China Postdoctoral Science Foundation (2020M683126).

\section{Data availability statement}

The data that supports the findings of this study are available from the corresponding author on request.

\section{Ethics approval and consent to participate}

The study was approved by the Ethics Committee (full name: Regional Ethics Committee of The First Affiliated Hospital of Jinzhou Medical University) to the Department of Surgery, Jinzhou Medical University.

\section{Consent for publication}




\section{References}

1. van den Bosch MHJ. Osteoarthritis year in review 2020: biology. Osteoarthritis Cartilage. 2021;29(2):143-50.

2. Peat G, Thomas MJ. Osteoarthritis year in review 2020: epidemiology \& therapy. Osteoarthritis Cartilage. 2021;29(2):180-9.

3. Whittaker JL, et al. Osteoarthritis year in review 2020: rehabilitation and outcomes. Osteoarthritis Cartilage. 2021;29(2):190-207.

4. Ouyang $\mathrm{Y}$, et al. Overexpression of SOX9 alleviates the progression of human osteoarthritis in vitro and in vivo. Drug Des Devel Ther. 2019;13:2833-42.

5. Vargas Negrin F, et al. [Treatment of patients with osteoarthritis]. Aten Primaria. 2014;46(Suppl 1):39-61.

6. Lane NE, Shidara K, Wise BL. Osteoarthritis year in review 2016: clinical. Osteoarthritis Cartilage. 2017;25(2):209-15.

7. Bannuru RR, et al. Therapeutic trajectory of hyaluronic acid versus corticosteroids in the treatment of knee osteoarthritis: a systematic review and meta-analysis. Arthritis Rheum. 2009;61(12):1704-11.

8. Jones T, Kelsberg G, Safranek S. FPIN's clinical inquiries: Intra-articular corticosteroid injections for osteoarthritis of the knee. Am Fam Physician. 2014;90(2):115-6.

9. Vandeweerd JM, et al. Effect of corticosteroids on articular cartilage: have animal studies said everything? Fundam Clin Pharmacol. 2015;29(5):427-38.

10. McAlindon TE, et al. Effect of Intra-articular Triamcinolone vs Saline on Knee Cartilage Volume and Pain in Patients With Knee Osteoarthritis: A Randomized Clinical Trial. JAMA. 2017;317(19):196775.

11. Zeng C, et al. Intra-articular corticosteroids and the risk of knee osteoarthritis progression: results from the Osteoarthritis Initiative. Osteoarthritis Cartilage. 2019;27(6):855-62.

12. Song $Y$, et al. Mesenchymal stem cells in knee osteoarthritis treatment: A systematic review and meta-analysis. J Orthop Translat. 2020;24:121-30.

13. Ha CW, et al. Intra-articular Mesenchymal Stem Cells in Osteoarthritis of the Knee: A Systematic Review of Clinical Outcomes and Evidence of Cartilage Repair. Arthroscopy. 2019;35(1):277-88 e2.

14. Harrell CR, et al. Mesenchymal stem cell-based therapy of osteoarthritis: Current knowledge and future perspectives. Biomed Pharmacother. 2019;109:2318-26.

15. Monteiro N, et al. Nanoparticle-based bioactive agent release systems for bone and cartilage tissue engineering. Regen Ther. 2015;1:109-18.

16. Wu HH, et al. Mesenchymal stem cell-based drug delivery strategy: from cells to biomimetic. J Control Release. 2019;294:102-13. 
17. Fang RH, et al. Cell Membrane Coating Nanotechnology Adv Mater. 2018;30(23):e1706759.

18. Ahn J, et al. Microfluidics in nanoparticle drug delivery; From synthesis to pre-clinical screening. Adv Drug Deliv Rev. 2018;128:29-53.

19. Cliffel DE, Turner BN, Huffman BJ. Nanoparticle-based biologic mimetics. Wiley Interdiscip Rev Nanomed Nanobiotechnol. 2009;1(1):47-59.

20. Richardson SM, et al. Mesenchymal stem cells in regenerative medicine: Focus on articular cartilage and intervertebral disc regeneration. Methods. 2016;99:69-80.

21. $\mathrm{Ng} \mathrm{J}$, et al. Stem cell-directed therapies for osteoarthritis: The promise and the practice. Stem Cells. 2020;38(4):477-86.

22. Lu J, et al. Increased recruitment of endogenous stem cells and chondrogenic differentiation by a composite scaffold containing bone marrow homing peptide for cartilage regeneration. Theranostics. 2018;8(18):5039-58.

23. Neybecker $P$, et al. In vitro and in vivo potentialities for cartilage repair from human advanced knee osteoarthritis synovial fluid-derived mesenchymal stem cells. Stem Cell Res Ther. 2018;9(1):329.

24. Hermida-Gomez T, et al. Quantification of cells expressing mesenchymal stem cell markers in healthy and osteoarthritic synovial membranes. J Rheumatol. 2011;38(2):339-49.

25. Tan SSH, et al., Mesenchymal Stem Cell Exosomes for Cartilage Regeneration: A Systematic Review of Preclinical In Vivo Studies. Tissue Eng Part B Rev, 2021. 27(1): p. 1-13.

26. Greening DW, et al. A protocol for exosome isolation and characterization: evaluation of ultracentrifugation, density-gradient separation, and immunoaffinity capture methods. Methods Mol Biol. 2015;1295:179-209.

27. Koh YQ, et al. Exosome enrichment by ultracentrifugation and size exclusion chromatography. Front Biosci (Landmark Ed). 2018;23:865-74.

28. Li R, et al. Route to Rheumatoid Arthritis by Macrophage-Derived Microvesicle-Coated Nanoparticles. Nano Lett. 2019;19(1):124-34.

29. Fang RH, et al. Quick synthesis of lipid-polymer hybrid nanoparticles with low polydispersity using a single-step sonication method. Langmuir. 2010;26(22):16958-62.

30. Uson J, et al. EULAR recommendations for intra-articular therapies. Ann Rheum Dis. 2021;80(10):1299-305.

31. Zhang W, et al. EULAR evidence based recommendations for the management of hand osteoarthritis: report of a Task Force of the EULAR Standing Committee for International Clinical Studies Including Therapeutics (ESCISIT). Ann Rheum Dis. 2007;66(3):377-88.

32. Zhang R, et al. Mesenchymal stem cell related therapies for cartilage lesions and osteoarthritis. Am J Transl Res. 2019;11(10):6275-89.

33. Doyle LM, Wang MZ. Overview of Extracellular Vesicles, Their Origin, Composition, Purpose, and Methods for Exosome Isolation and Analysis. Cells, 2019. 8(7). 
34. Zhang Y, et al. Exosome: A Review of Its Classification, Isolation Techniques, Storage, Diagnostic and Targeted Therapy Applications. Int J Nanomedicine. 2020;15:6917-34.

35. Morath I, Hartmann TN, Orian-Rousseau V, CD44: More than a mere stem cell marker. Int J Biochem Cell Biol, 2016. 81(Pt A): p. 166-173.

36. Beier F. Cell-cycle control and the cartilage growth plate. J Cell Physiol. 2005;202(1):1-8.

37. Griffin TM, Scanzello CR. Innate inflammation and synovial macrophages in osteoarthritis pathophysiology. Clin Exp Rheumatol. 2019;37(5):57-63.. . Suppl 120.

38. Wang $X$, et al. The importance of synovial inflammation in osteoarthritis: current evidence from imaging assessments and clinical trials. Osteoarthritis Cartilage. 2018;26(2):165-74.

39. Fernandes TL, et al. Macrophage: A Potential Target on Cartilage Regeneration. Front Immunol. 2020;11:111.

40. Ma X, et al., The Roles of FoxO Transcription Factors in Regulation of Bone Cells Function. Int J Mol Sci, 2020. 21(3).

41. Akasaki Y, et al. Dysregulated FOXO transcription factors in articular cartilage in aging and osteoarthritis. Osteoarthritis Cartilage. 2014;22(1):162-70.

\section{Figures}


A
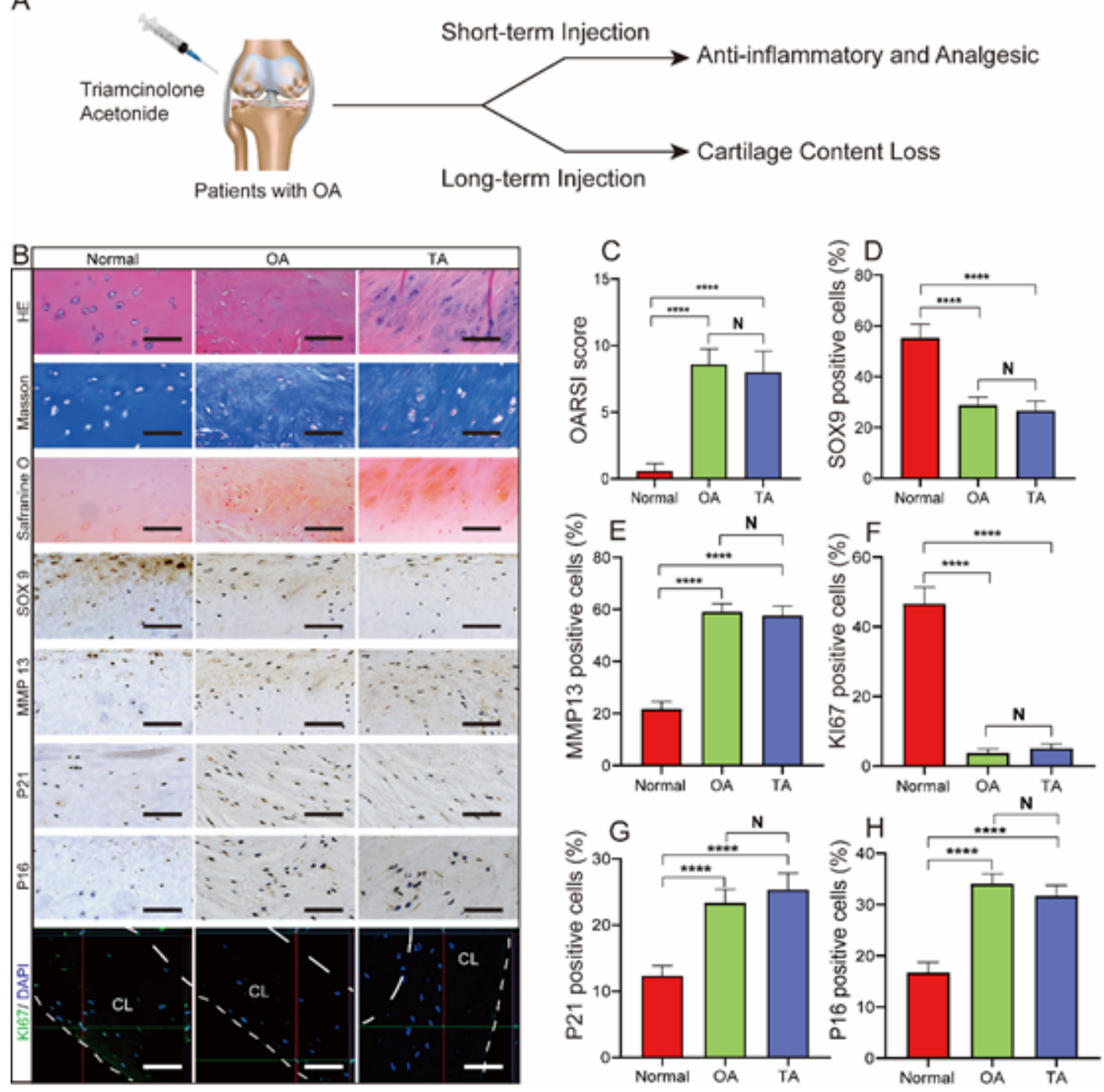

Figure 1

Pathological detection of TA IA in patients with OA. 
A
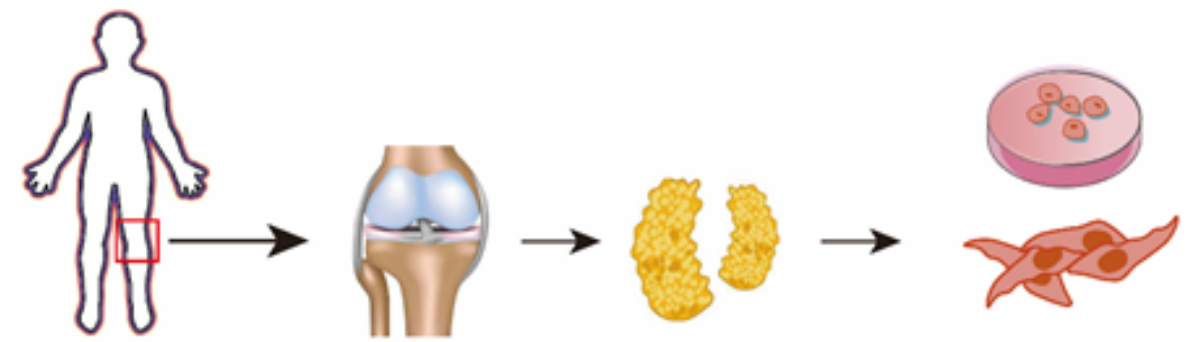

Healthy human synovial tissue

CD90 positive synovial MSCs

B

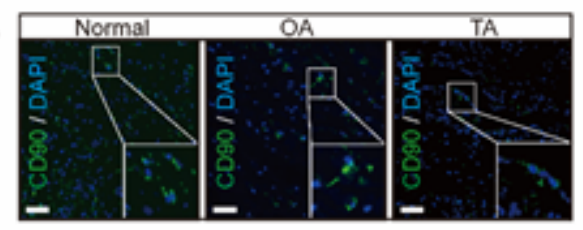

C

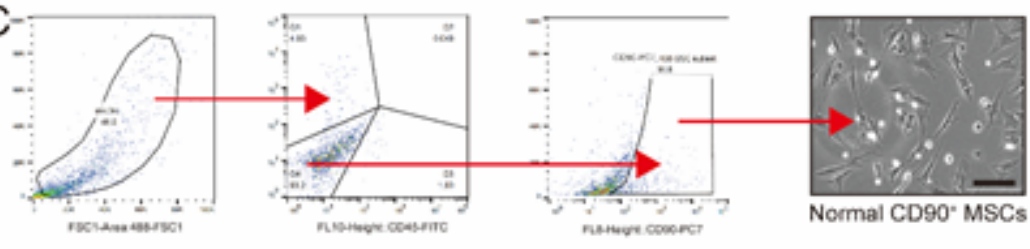

D

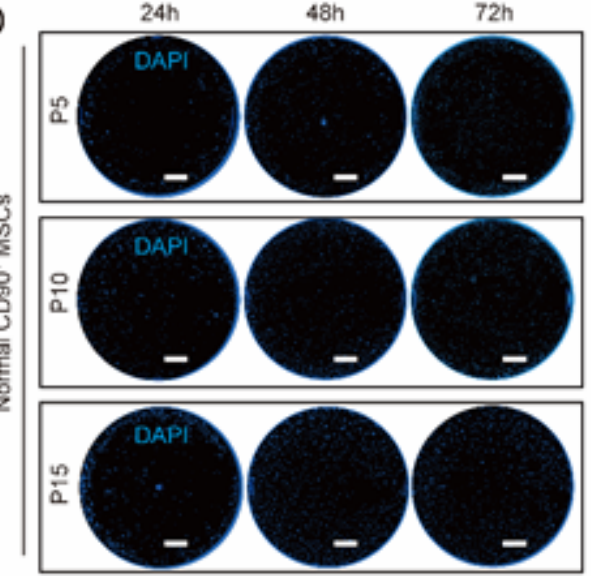

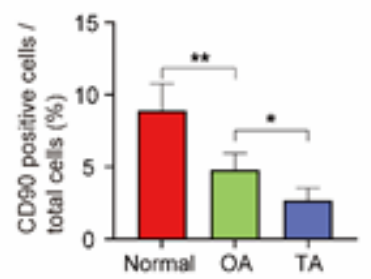

E

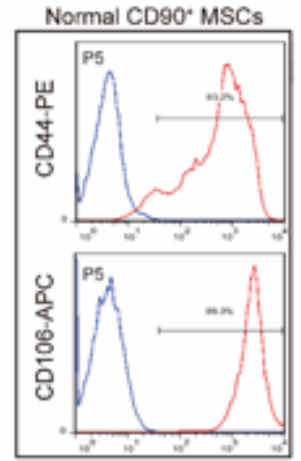

$\mathrm{F}$

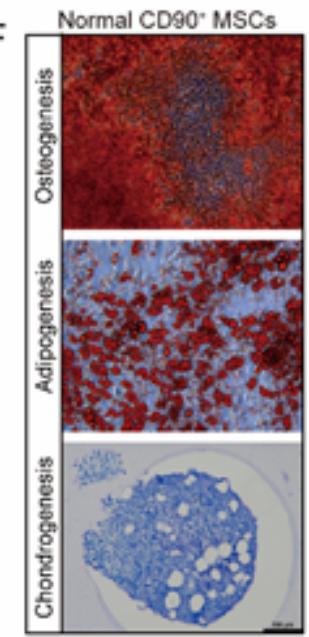

Figure 2

Extraction and identification of CD90+ MSCs from healthy human synovium. 
A
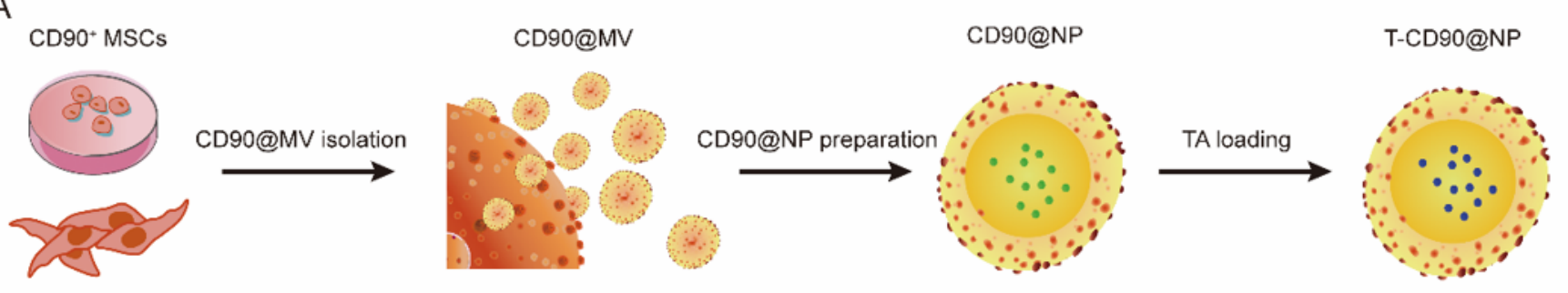

B

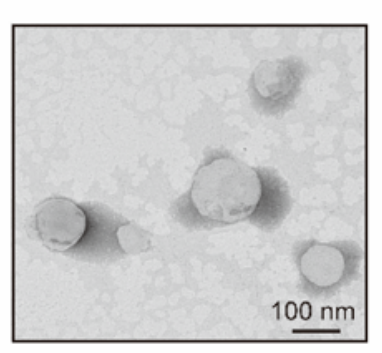

E

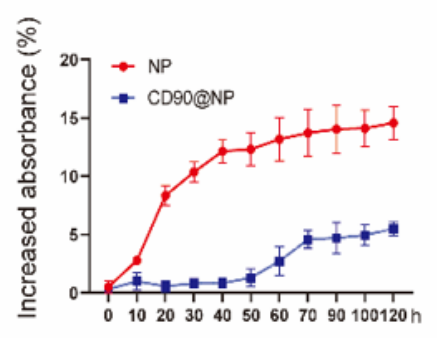

C

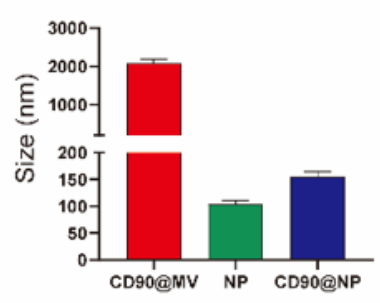

F

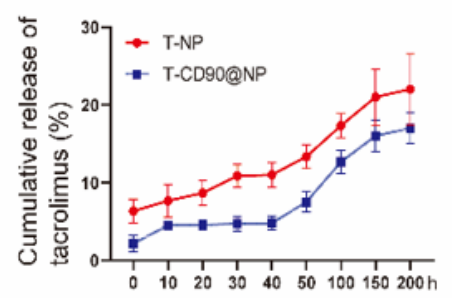

D
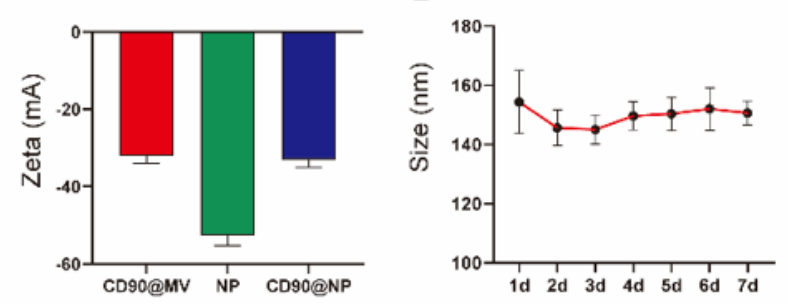

G

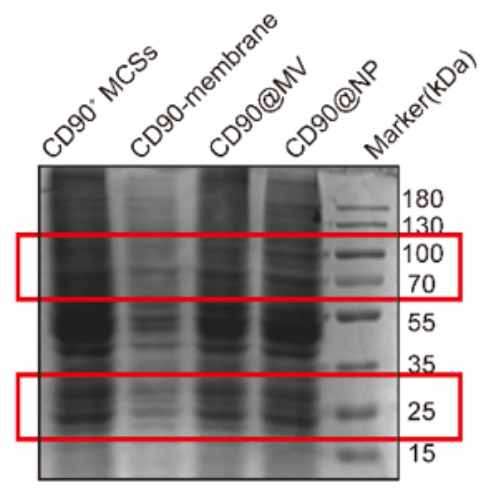

$\mathrm{H}$

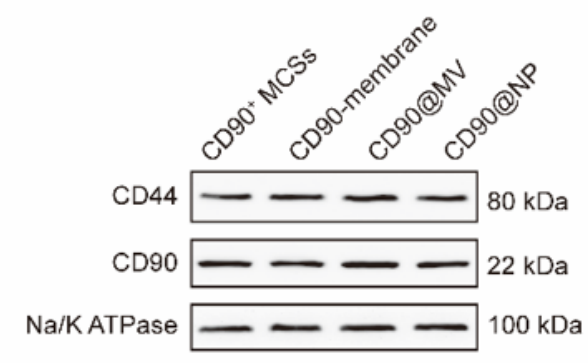

I

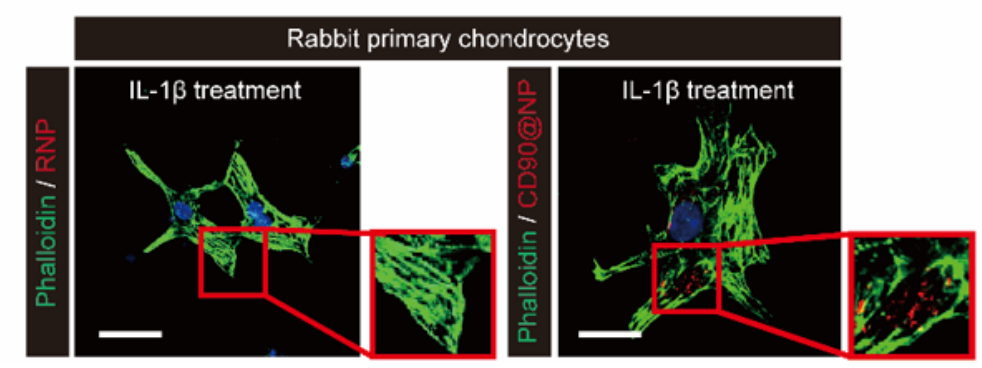

Figure 3

Characterization of CD90@NP. 

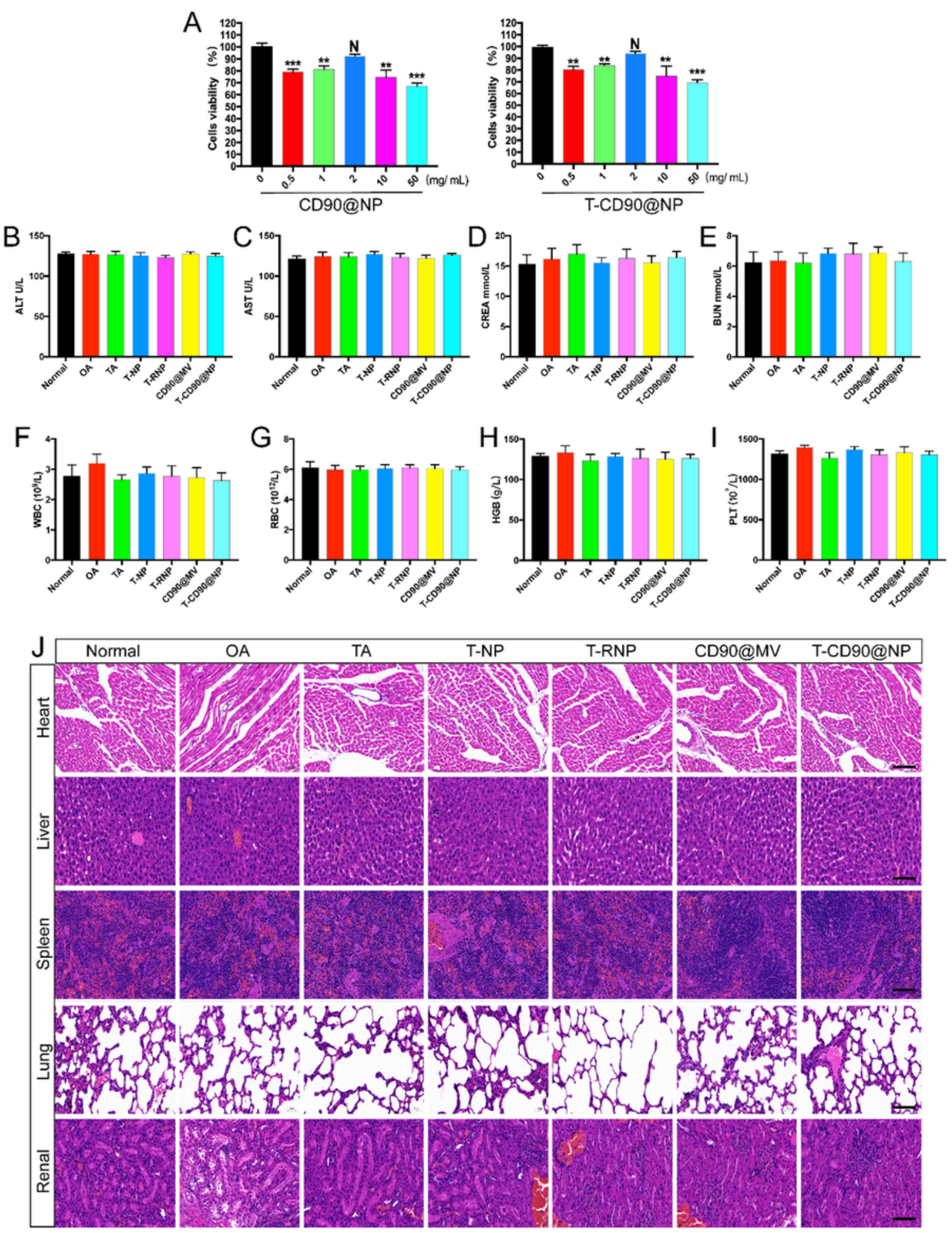

Figure 4

Preliminary toxicity study in vivo and in vitro. 
A
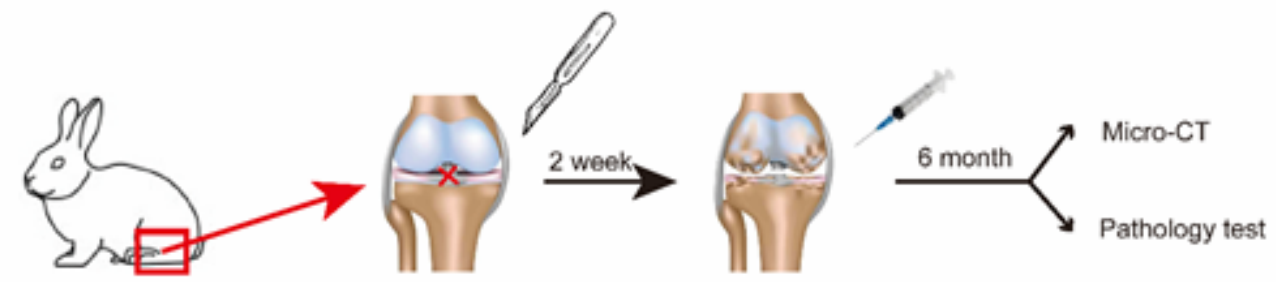

6-month-old Female Rabbit

ACLT Model (OA)

TCD90@NPIA
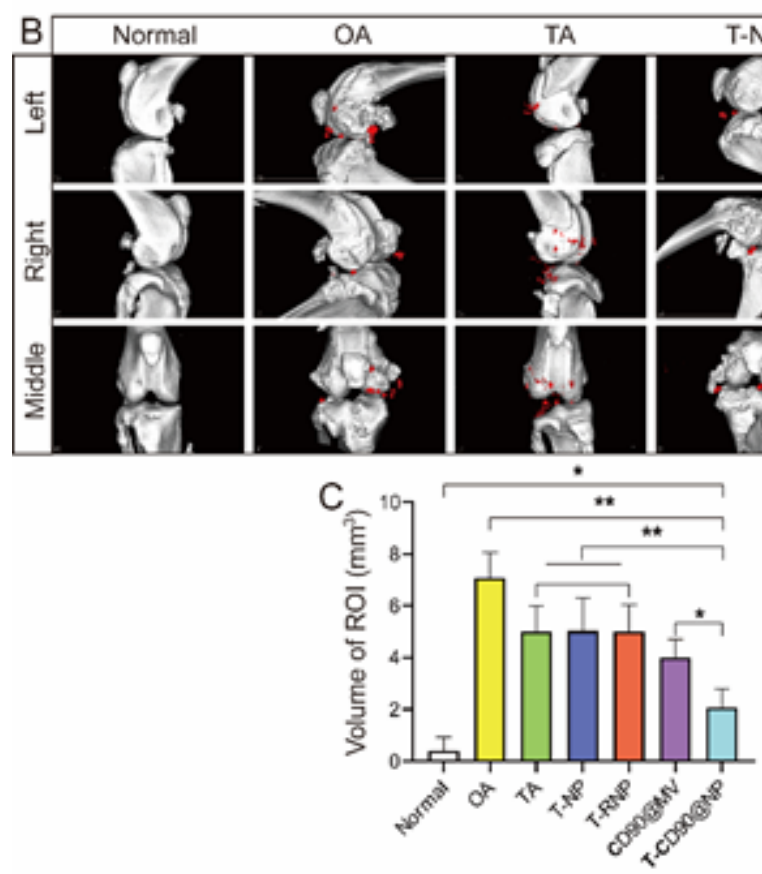

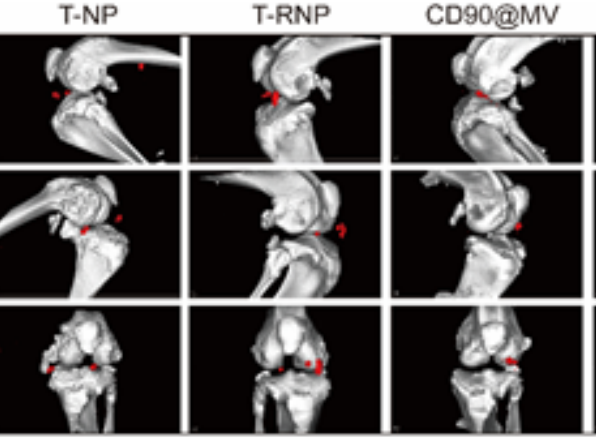

D

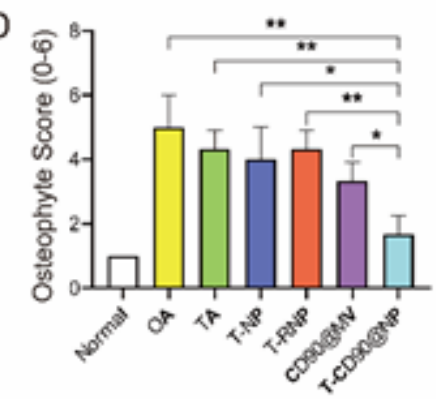

Figure 5

Micro-CT detection of the rabbit knee joint. 

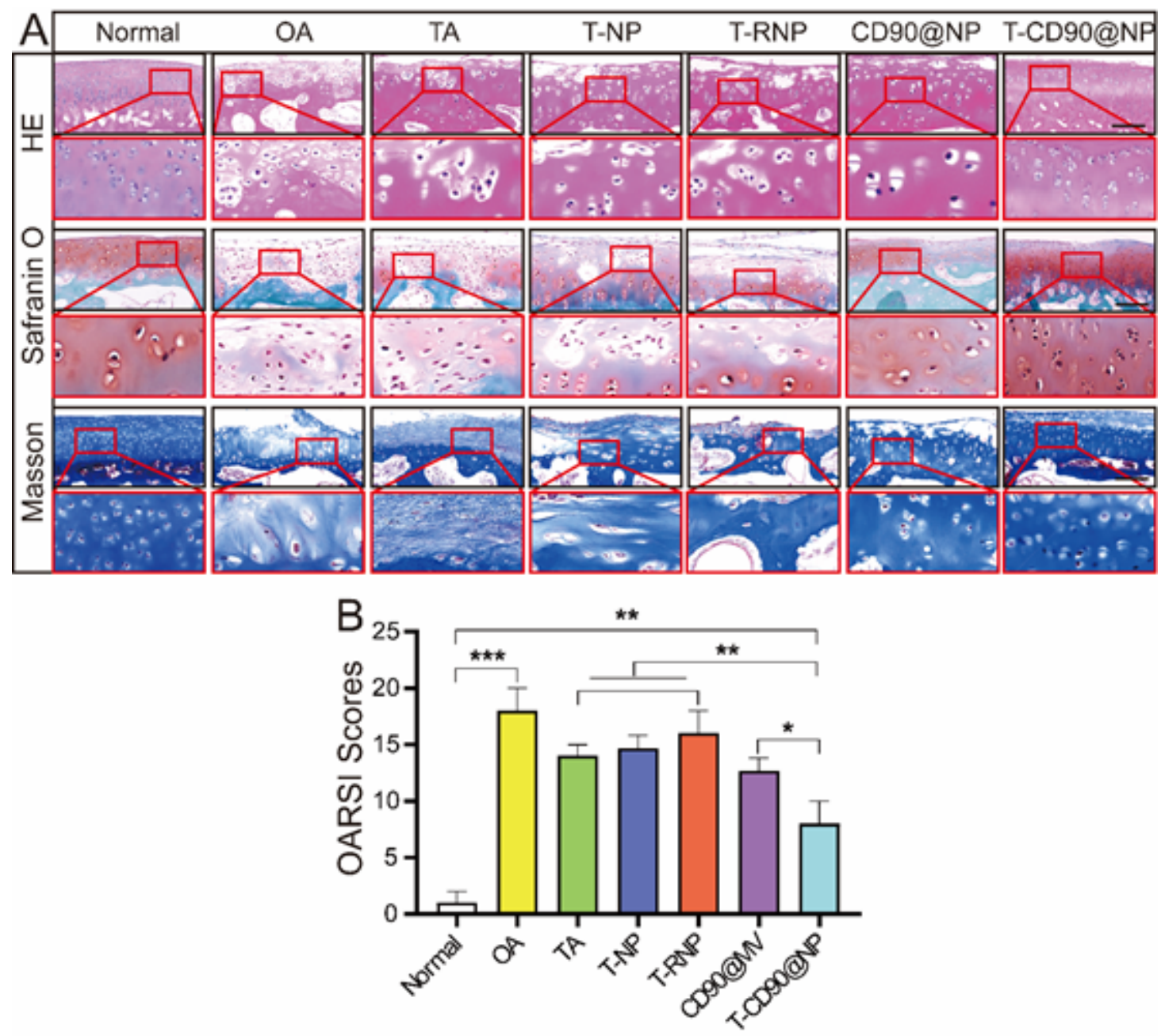

Figure 6

Pathological examination of the rabbit knee joint. 

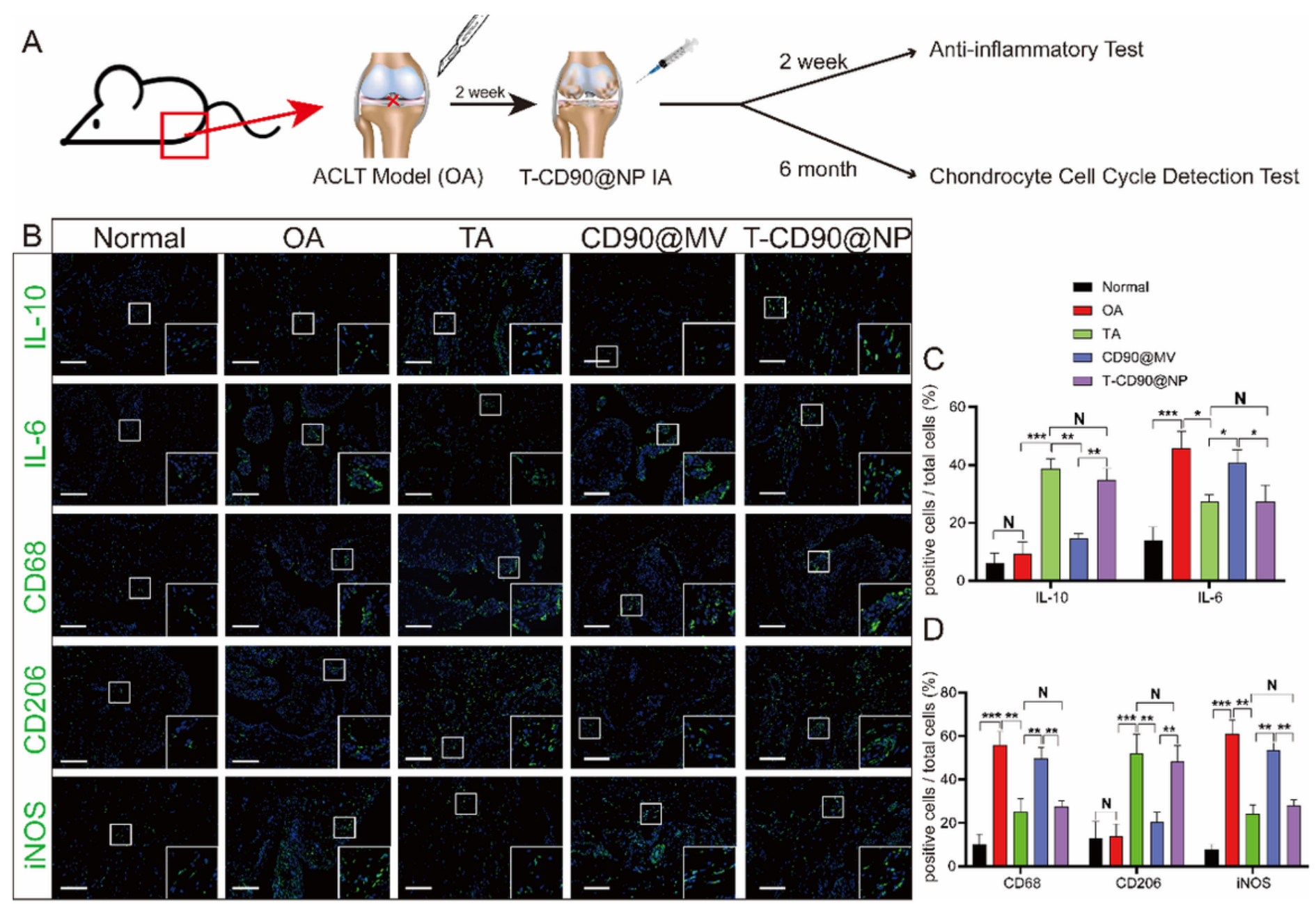

Figure 7

Detection of synovium inflammation levels in rats. 

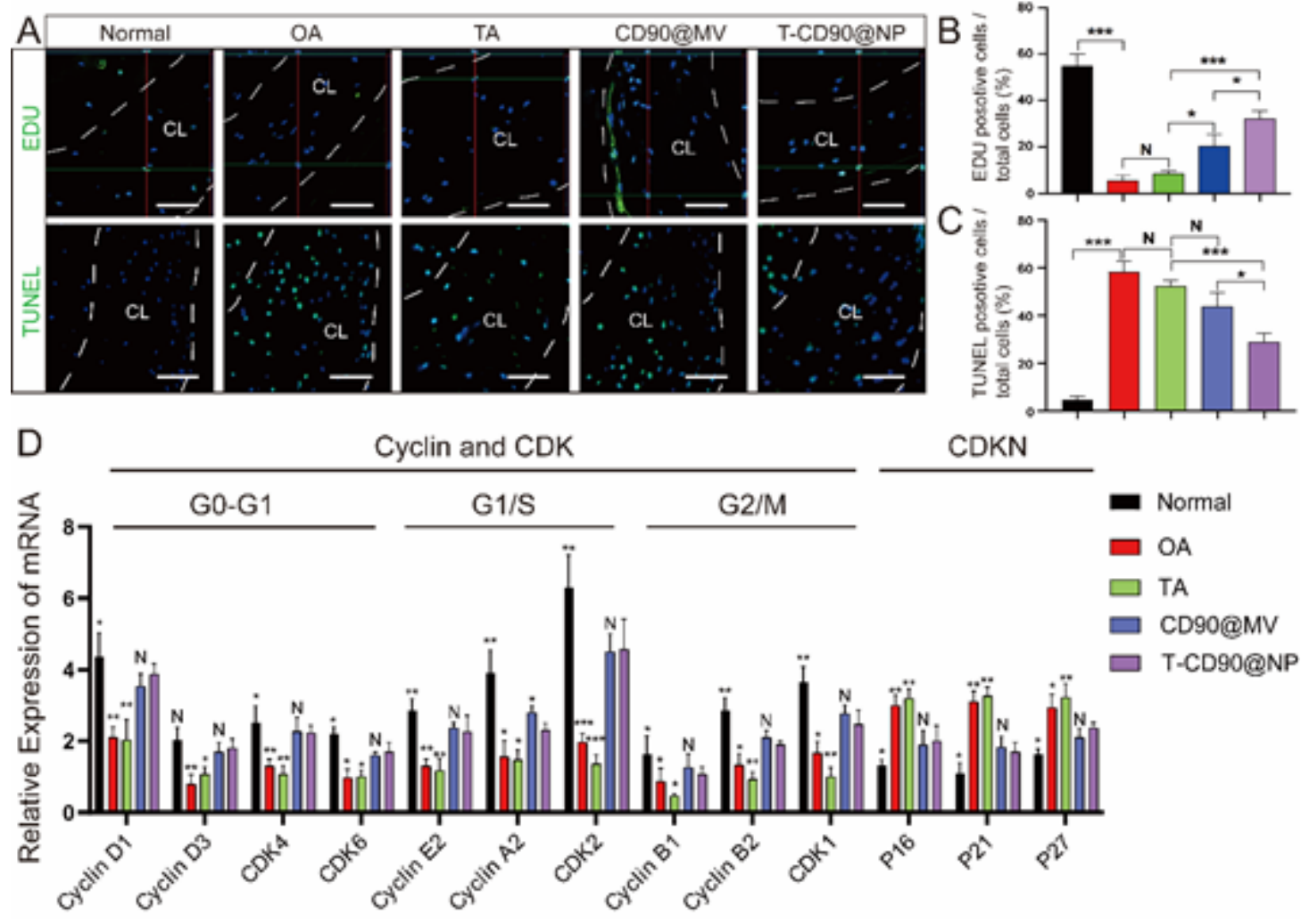

Figure 8

Regeneration and apoptosis of knee articular cartilage in rats. 
A

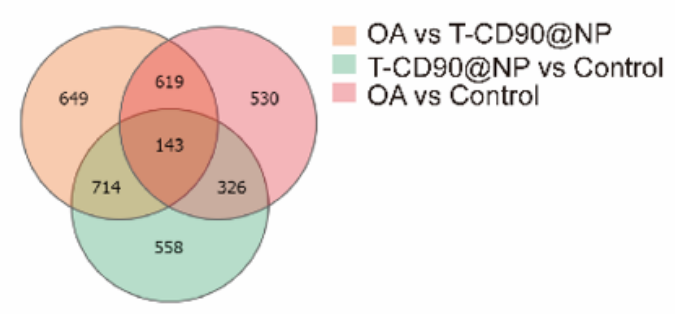

C

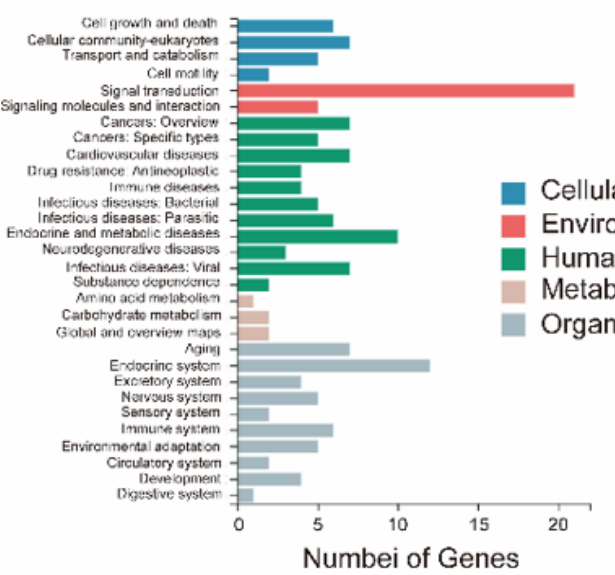

B

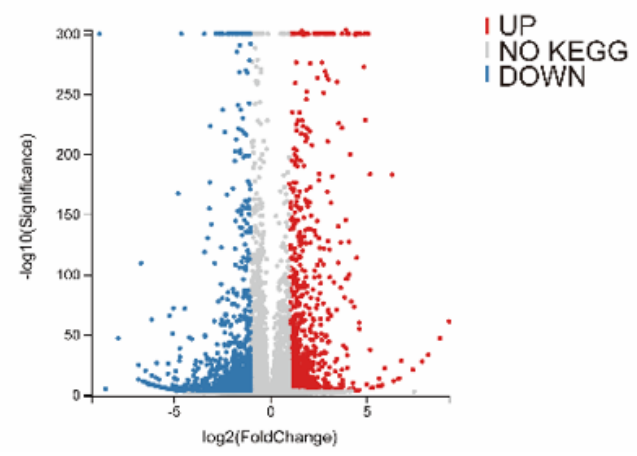

$E$

Cellular Processesh

Erocessing

Metabolism

rganismal Systems
D

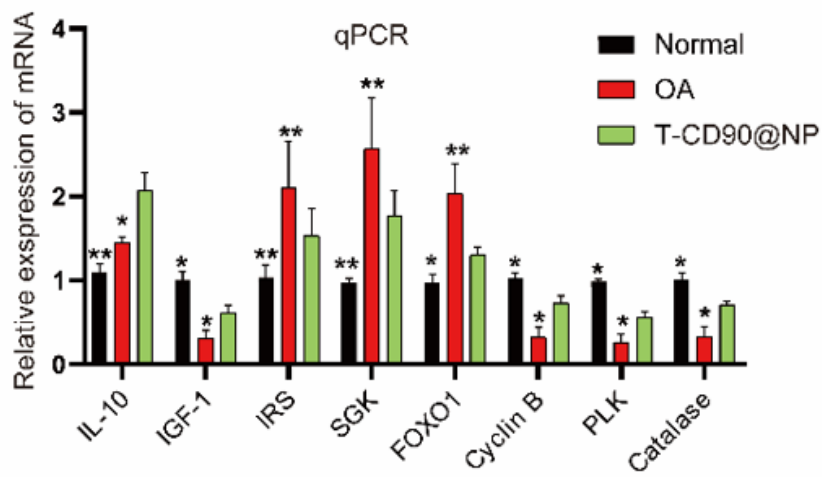

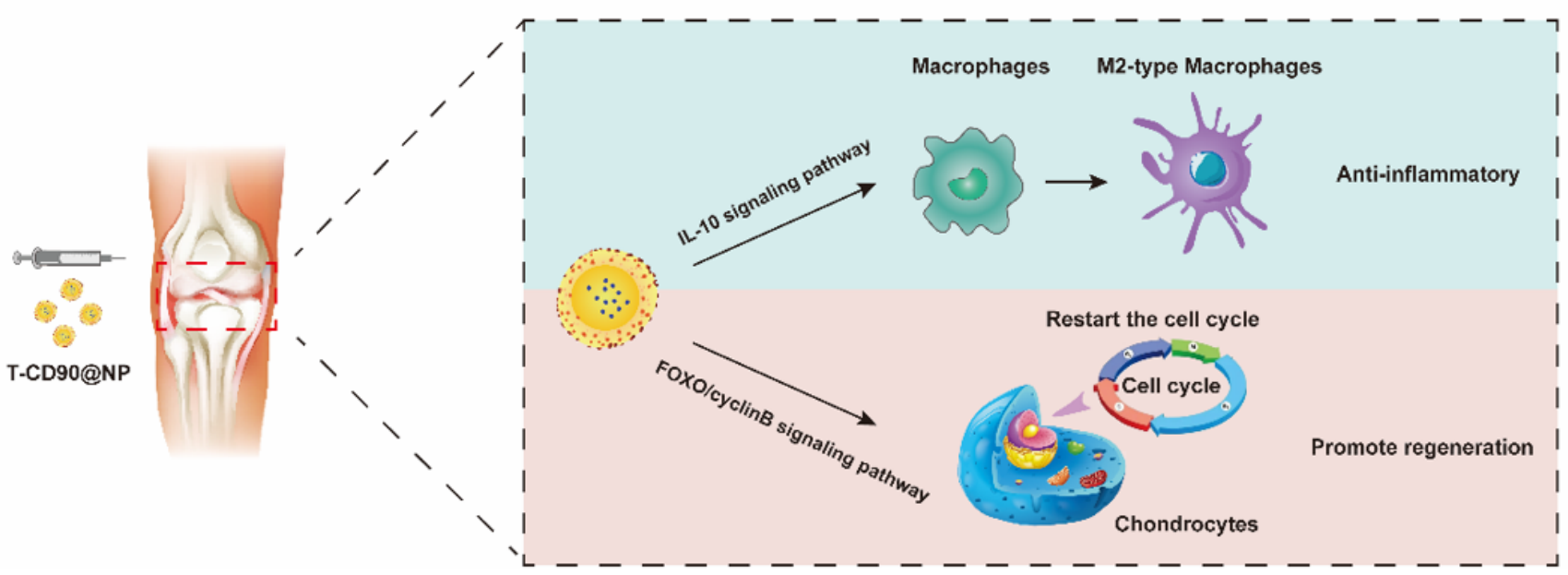

Figure 9

Regeneration and apoptosis of knee articular cartilage in rats.

\section{Supplementary Files}

This is a list of supplementary files associated with this preprint. Click to download. 
- Scheme1.png

- figs1.png

Page $30 / 30$ 